\title{
LE ROLE DU FACTEUR AUTOSOMAL D'EMPLUMEMENT DANS LA CROISSANGE EN POIDS CHEZ LES VOLAILLES
}

\author{
PAR \\ Ph. MERAT \\ Station de Recherches Avicoles, Jouy-en-Josas (S.-et-O.).
}

La vitesse d'emplumement du corps chez le poulet, est en général appréciée à vue. Elle peut dépendre du couple allélique $K, k$, lié au sexe, mais aussi d'un facteur autosomal, que Hu'T (I949) identifie à un gène unique.

On a plusieurs fois noté une liaison positive entre vitesse d'emplumement due au facteur " autosomal " et croissance en poids jusqu'à l'âge de 6 à I2 semaines.

Nous avons vérifié ce fait sur 4 populations différentes, mais en mesurant l'emplumement suivant la méthode plus précise décrite par MUELLER et al. (1952) d'après le nombre de rectrices qui ont déjà mué et ont été remplacées à 8 semaines (nombre compris entre o et 8). La mesure était faite seulement chez les porteurs de l'emplumement rapide lié au sexe (kk ou k-).

L'emplumement ainsi défini comportant 9 classes, on peut évaluer son coefficient de corrélation avec le poids à 8 semaines, ainsi que les deux coefficients de régression. Les résultats, concordant sur les 4 populations, indiquent une liaison positive plus marquée d'ailleurs chez les femelles que chez les mâles (tableau I) :

\section{TABLEAU I}

\begin{tabular}{|c|c|c|c|c|c|}
\hline & \multirow[b]{2}{*}{ Population } & \multicolumn{2}{|c|}{ Máles } & \multicolumn{2}{|c|}{ Femelles } \\
\hline & & $\begin{array}{l}\text { Corrélation } \\
\text { empl. } \times \text { poids } \\
\text { à } 8 \text { sem. }\end{array}$ & $\left\{\begin{array}{l}\text { Coef. de régression } \\
\frac{\text { poids à } 8 \text { s. (en (r.) }}{\text { emplumement }}\end{array}\right.$ & $\begin{array}{l}\text { Corrëlation } \\
\text { empl. } \times \text { poids } \\
\text { à } 8 \text { sem. }\end{array}$ & $\begin{array}{l}\text { Coef. de régression } \\
\text { poids à } 8 \text { s. (en g.) } \\
\text { cmplumement }\end{array}$ \\
\hline $\mathrm{T}$ & 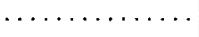 & $+0,248$ & $+\mathrm{I} 5, \mathrm{I}$ & $+c, 295$ & $+7,0$ \\
\hline 2 & $\ldots$ & $+0,182$ & $+10,5$ & $+0,277$ & $+12, I$ \\
\hline 3 & $\ldots \ldots \ldots \ldots$ & $+0,155$ & $+6,6$ & $+0,283$ & $+10,5$ \\
\hline 4 & $\ldots \ldots \ldots \ldots \ldots$ & $+0,105$ & $+4,3$ & $+0,167$ & $+8,0$ \\
\hline
\end{tabular}

Un autre fait, non observé jusqu'ici, est la variabilité moindre du poids chez les animaux bien emplumés, surtout chez les femelles (tableau II). 
La liaison positive entre croissance et emplumement "autosomal" provient donc au moins en partie d'une proportion plus faible d'animaux à croissance retardée parmi les poulets bien emplumés.

TABLEAU II

Variance (intra-populations et corrigée pour la date d'éclosion) pour le poids à 8 semaines (en g.) suivant l'emplumement (populations groupées).

\begin{tabular}{|c|c|c|c|c|c|c|}
\hline & \multicolumn{2}{|c|}{ o, I ou 2 rectrices remplacées } & \multicolumn{2}{|c|}{3,4 ou, 5 rectrices remplacées } & \multicolumn{2}{|c|}{6,7 ou 8 rectrices remplacées } \\
\hline & $\begin{array}{l}\text { Variance } \\
\text { du poids }\end{array}$ & $\begin{array}{l}\text { Degrés } \\
\text { de liberté }\end{array}$ & $\begin{array}{l}\text { Variance } \\
\text { du poids }\end{array}$ & $\begin{array}{l}\text { Deurés } \\
\text { de liberté }\end{array}$ & $\begin{array}{l}\text { Variance } \\
\text { du poids }\end{array}$ & $\begin{array}{l}\text { Desrrés } \\
\text { de liberté }\end{array}$ \\
\hline $\begin{array}{l}0 \\
\phi \\
\phi\end{array}$ & $\begin{array}{l}\text { I } 2760 \\
\text { II } 6,30\end{array}$ & $\begin{array}{l}855 \\
652\end{array}$ & $\begin{array}{r}11060 \\
8510\end{array}$ & $\begin{array}{r}998 \\
1.343\end{array}$ & $\begin{array}{r}10,550 \\
7640\end{array}$ & $\begin{array}{r}185 \\
+42\end{array}$ \\
\hline
\end{tabular}

Ceci présente un intérêt pour la sélection en vue d'une croissance homogène en même temps que d'un emplumement précoce, car l'emplumement ainsi mesuré semble un caractère fortement héritable. Ies données supplémentaires doivent montrer si sa réalisation dépend d'un gène majeur unique, comme dans les observations de HuT'T.

L'emplumement précoce autosomal s'accompagne également chez les animaux étudiés d'une maturité sexuelle légèrement plus hâtive, et d'une mortalité peut-être moindre entre l'âge de $I 2$ semaines et le 3 I décembre suivant, observation qui semble également nouvelle et pourrait faire entrer aussi en ligne de compte ce facteur dans la sélection pour la ponte.

\section{REFFERENCES BIBLIOGRAPHIQUES}

Hutr (F. B.). - Genetics of the fowl. Me Graw Hill Book Co, N. Y. - I949. Mueliter (C. D.), Moultrie (F.) et Plumart (P. E.). - Classification of feathering in broiler stock. Kansas Agr. Exp. Sta. Bull. n 72, I952. 